\title{
Review Article \\ Clinical Significance of FGF-23 in Patients with CKD
}

\author{
Domenico Russo and Yuri Battaglia \\ Department of Nephrology, School of Medicine, University Federico II, Via Pansini 5, 80131 Napoli, Italy \\ Correspondence should be addressed to Domenico Russo, domenicorusso51@hotmail.com
}

Received 22 December 2010; Accepted 24 February 2011

Academic Editor: Angela Wang

Copyright ( 12011 D. Russo and Y. Battaglia. This is an open access article distributed under the Creative Commons Attribution License, which permits unrestricted use, distribution, and reproduction in any medium, provided the original work is properly cited.

FGF23 is a bone-derived hormone that plays an important role in the regulation of phosphate and 1,25-dihydroxy vitamin D metabolism. FGF23 principally acts in the kidney to induce urinary phosphate excretion and suppress 1,25-dihydroxyvitamin D synthesis in the presence of FGF receptor 1 (FGFR1) and its coreceptor Klotho. In patients with chronic kidney disease (CKD), circulating FGF23 levels are progressively increased to compensate for persistent phosphate retention, but this results in reduced renal production of 1,25-dihydroxyvitamin D and leads to hypersecretion of parathyroid hormone. Furthermore, FGF23 is associated with vascular dysfunction, atherosclerosis, and left ventricular hypertrophy. This paper summarizes the role of FGF23 in the pathogenesis of mineral, bone, and cadiovascular disorders in CKD.

\section{Introduction}

Patients with chronic kidney disease (CKD) are at increased risk for cardiovascular events compared with individuals with normal kidney function [1]. In addition to traditional cardiovascular risk factors, disturbances in calciumphosphate metabolism are regarded as strong contributing factors of higher cardiovascular mortality in CKD patients $[2,3]$. Indeed, elevated serum phosphate, low calcitriol, and high PTH levels represent the classical triad that leads to secondary hyperparathyroidism, each factor being independently associated with cardiovascular events and mortality in patients with CKD $[4,5]$.

Recently, the phosphaturic hormone, fibroblast growth factor (FGF-23), is overpoweringly entered into the traditional pathophysiological scheme of secondary hyperparathyroidism.

\section{Structure of FGF-23}

Seven known subfamilies of human FGFs have been defined $[6,7]$. The FGF-19 subfamily is composed of three proteins-FGF-19, FGF-21, and FGF-23-which exert diverse physiological functions. FGF-23 is a central regulator of phosphate homeostasis and calcitriol blood levels; FGF-19 inhibits the expression of enzyme cholesterol 7-ahydroxylase (CYP7A1), which is the first and rate-limiting step in bile acid synthesis [8]; FGF-21 stimulates insulinindependent glucose uptake in adipocytes and lowers triglycerides [9]. Interestingly, FGF-19, FGF-21, and FGF-23 contain a disulfide bond that is absent in most other subfamilies. This may explain why FGF-23 can be distributed in the bloodstream to mediate its functions.

FGF-23 is a 251-amino acid protein (MW $26 \mathrm{kDa}$ ) synthesized and secreted by bone cells, mainly osteoblast [10]. It is composed of an amino-terminal signal peptide (residues 1-24), followed by an "FGF-like sequence" (residues 25-180) and a carboxyl-terminal extended sequence (residues 181251) that is unique compared with other members of the FGF family [7]. The half-life of intact FGF-23 in the circulation of healthy individuals has been estimated to be 58 min [11]. FGF-23 exerts its biological effects through activation of FGF receptors (FGF-Rs); this activation is Klotho dependent as a Klotho/FGF-R complex binds to FGF-23 with higher affinity than does FGF-R or Klotho alone [12]. Klotho is a 130$\mathrm{kDa}$ transmembrane b-glucuronidase capable of hydrolyzing 
steroid b-glucoronides. It was named after Klotho, one of the Moirae (the fates) in Greek mythology who spun the thread of life from her distaff onto her spindle; indeed, Klothodeficient mice manifest a syndrome resembling accelerated human aging and extensive atherosclerosis. Because FGF-23/mice show similar phenotypes to Klotho/mice, a common signaling pathway has been postulated $[13,14]$. Klotho gene expression was detected in cells of the renal tubule, parathyroid, and choroid plexus. Importantly, renal Klotho expression is largely confined to the distal tubules, which is also the site for initial FGF-23 binding and signaling $[15,16]$. However, renal phosphate reabsorption mainly occurs in the proximal tubules, and it is currently unknown how FGF-23 signaling in the distal tubule translates into decreased phosphate reabsorption in the proximal tubules.

\section{Physiological Functions of FGF-23}

Renal phosphate excretion is physiologically regulated mainly by proximal tubular cells, which express both Na/Pi Type II and $\mathrm{Na} / \mathrm{Pi}$ IIc cotransporters at their apical membrane [17].

FGF-23 reduces the action of both cotransporters; in addition, it may inhibit gastrointestinal phosphate absorption by reducing intestinal $\mathrm{Na} / \mathrm{Pi}$ IIb cotransporter activity in a vitamin D-dependent manner [18].

The principal physiological stimuli for increased FGF-23 expression both in vitro and in vivo are 1,25(OH)2D3 and high dietary phosphate intake [19-22]. Persistent hyperphosphataemia is an effective trigger for FGF-23, while rapid changes in serum phosphate concentrations may not induce an acute increment in serum FGF-23 levels [23]. It is therefore possible that FGF-23 responds to the net phosphate balance rather than to the serum phosphate level, but experimental data supporting this hypothesis are scarce.

\section{FGF-23, PTH, and Calcitriol}

FGF-23, PTH, and calcitriol may influence each other in opposite manner. FGF-R and Klotho are expressed in parathyroid glands; FGF-23 might decrease PTH mRNA transcription [24]. FGF-23 activity is not dependent on PTH, as the phosphaturic effects of FGF-23 are maintained in animals after parathyroidectomy [25]. Conversely, PTH may stimulate FGF-23 secretion by osteoblast, as FGF-23 levels are increased in rodents with primary HPT, which may be reversed by parathyroidectomy [26]. In rodents, injection of recombinant FGF-23 reduces calcitriol levels within hours by decreasing renal expression of 1a-hydroxylase (CYP27B1) and increasing the expression of 24-hydroxylase (CYP24A1), which controls calcitriol degradation [25]. Conversely, calcitriol itself stimulates FGF-23 generation by binding to a vitamin $\mathrm{D}$ response region in the FGF-23 gene promoter [27].

\section{FGF-23 in Subjects with Intact Renal Function}

The main physiological role of FGF-23 in healthy subjects is to regulate urinary phosphate excretion to maintain stable serum phosphate levels. However, no correlation between FGF-23 and serum phosphate levels has been found in individuals without overt renal disease [28, 29]. Possible explanation for this finding is that most studies which found no significant change in FGF-23 levels were smaller, and phosphate loading was restricted to a maximum of 3 days [21].

\section{FGF-23 23 in Subjects with CKD}

In CKD, circulating FGF-23 levels gradually increase with declining renal function such that by the time patients reach end-stage renal disease, FGF23 levels can be up to 1000-fold above the normal range [30]. The increase in FGF-23 begins at a very early stage of CKD as a physiological compensation to stabilize serum phosphate levels as the number of intact nephrons declines [28, 30-32]. In contrast, it was hypothesized that increased FGF-23 levels in CKD result primarily from decreased renal clearance [31]. However, there is no increase in the accumulation of degraded FGF-23 in advanced CKD. It is also likely that FGF-23 levels depend on an increased secretion due to an end-organ resistance to the phosphaturic stimulus of FGF-23 because of a deficiency of the necessary Klotho cofactor $[33,34]$. Other potential explanations for the early rise in FGF-23 could be the release of unidentified FGF-23 stimulatory factors or loss of a negative feedback factor(s) that normally suppress FGF-23, by the failing kidney.

\section{FGF-23, Mortality, and Cardiovascular End Points}

Since alterations in mineral metabolism are associated with increased cardiovascular risk in CKD, it is plausible that FGF-23 is directly involved in it. Indeed, in patients starting hemodialysis, higher FGF-23 levels were strongly associated with increased risk of 1-year mortality both in crude- and multivariate-adjusted models, with the highest FGF-23 quartile reaching a nearly 6-fold higher risk than the lowest [35]. In addition, in this population, FGF-23 was stronger predictor of mortality than the serum phosphate level. Importantly, FGF23 did not associate to mortality in patients within the highest quartile of serum phosphate $(>1.77 \mathrm{mmol} / \mathrm{L})$, suggesting that the prognostic value of FGF23 is blunted in the presence of severe hyperphosphatemia.

The role of FGF-23 on cardiovascular or renal end points but not on mortality has been evaluated in patients with CKD not on dialysis. In 227 diabetic patients with CKD stages 1-4, the progression of renal disease was assessed [32]. It was found that FGF-23 but not serum phosphate levels was significant independent predictor of CKD progression, defined as doubling of serum creatinine and/or terminal renal failure. In a cross-sectional study, a large cohort of 
men and women in CKD stage 2 with a mean eGFR of $73 \mathrm{~mL} / \mathrm{min} / 1.73 \mathrm{~m}^{2}$ was evaluated. In this cohort, that represented a valuable model of healthy individuals and early CKD, it was observed that higher FGF-23 was linked to several dynamic measurements of vascular function, including arterial stiffness measured by pulse wave velocity and endothelial dysfunction measured by an invasive forearm technique [36] in both crude- and multivariate-adjusted models. A subgroup of this population underwent a novel technique named whole-body magnetic resonance imaging angiography, which provides information about the degree of arterial stenosis as a surrogate marker of atherosclerosis in all major vascular territories. Higher FGF-23 level was associated with higher atherosclerosis score [37]. It is important to underline that FGF-23 in some studies has been linked to peripheral vascular calcification and/or coronary artery calcification score, whereas other reports have failed to show such an association $[29,38-41]$. On this regard, it has been speculated that FGF-23 could function as a local inhibitor of vascular calcification; FGF-23 inhibits calcification in vascular smooth muscle cells in vitro; this inhibitory effect is strengthened in an inflamed setting, which is often present in CKD patients [42]. Given the osteogenic transformation of vascular smooth muscle cells that occurs in atherosclerotic plaques, it is possible that FGF-23 may be locally expressed in the cardiovascular system. But the presence of FGF-23 in the heart or aorta has not been demonstrated yet [43]. It is currently thought that, at least in early CKD, FGF-23 indirectly contributes to decreased vascular calcification through maintaining a normal serum phosphate level. Finally, the relation between FGF-23 and left ventricular hypertrophy has been evaluated, that is another strong cardiovascular risk factor in CKD. This issue is clinically relevant because other members of the FGF family have been implicated in the pathogenesis of myocardial hypertrophy. Serum FGF23 was positively associated with left ventricular mass index and increased risk of having left ventricular hypertrophy. In particular, these associations were found in the highest FGF-23 tertile $(>48 \mathrm{pg} / \mathrm{mL})$ and were strengthened when restricted to subjects with eGFR $<60 \mathrm{~mL} / \mathrm{min} / 1.73 \mathrm{~m} 2$ [35]. It is worth noticing that the associations between FGF23 , vascular dysfunction, atherosclerosis, and left ventricular hypertrophy were all progressively strengthened in patients with a lower eGFR despite normal phosphate levels. This finding supports the hypothesis that FGF-23 may reveal information about phosphate-related toxicity that cannot be obtained by measurements of serum phosphate.

\section{Open Questions}

The mechanism by which FGF-23 increases cardiovascular events and mortality is still unclear. Thus, it is debated whether FGF-23 is merely a marker of disturbed calciumphosphate metabolism, or it exerts its undesirable effects by lowering vitamin D levels. Indeed, the correlation of FGF23 levels with serum phosphate in CKD patients [31, 32, 44, 45 ] and the association of hyperphosphatemia with adverse outcome in these patients $[4,5,46-52]$ may suggest that negative effects of FGF-23 on survival are the mirror of the negative effects of serum phosphorus. Alternatively, FGF23 may influence outcomes by inducing hypovitaminosis D suppressing 1a-hydroxylase with subsequent reduction in calcitriol secretion. Vitamin D deficiency is a nontraditional cardiovascular risk factor in CKD [53-55]. However, available data seem to exclude an ancillary role for FGF23 as mirror of serum phosphorus because adverse effects associated with high FGF-23 levels remained statistically significant after adjustment for phosphate, calcium, and PTH levels [32]. On the same way, adverse effects associated with high FGF-23 levels remained statistically significant after adjustment for vitamin D levels $[35,36]$. In addition, FGF23 has recently been shown to antagonize some effects of vitamin D in vitro; in a cell culture model, vitamin D induced cell apoptosis, whereas FGF-23 and Klotho induced cell proliferation [56]. Therefore, some hypotheses have been proposed. It has been hypothesized that FGF-23 at very high serum concentrations (as observed in CKD patients) may exert certain nonspecific and presumably adverse effects through low-affinity, Klotho-independent binding to FGF-R, for example, on endothelial cells [57].

\section{Conclusion}

FGF-23 is a regulator of calcium-phosphate metabolism. In clinical trials, elevated FGF-23 levels were independently associated with faster progression of CKD, therapy-resistant secondary hyperparathyroidism, left ventricular hypertrophy, and increased cardiovascular mortality in dialysis patients. However, FGF-23 is not just a marker of the derangements of calcium-phosphate metabolism in CKD, but rather a relevant factor responsible for the inception of secondary hyperparathyroidism and for cardiovascular morbidity and mortality. Thus, FGF-23 could represent a promising therapeutic target that might improve the fatal prognosis of patients with CKD.

\section{Disclosure}

The authors declare that the content of this paper has not been published elsewhere and is not currently under consideration by another journal published by SAGE-Hindawi or any other publisher. The paper's publication has been approved by all the other coauthors.

\section{References}

[1] R. N. Foley, P. S. Parfrey, and M. J. Sarnak, "Clinical epidemiology of cardiovascular disease in chronic renal disease," American Journal of Kidney Diseases, vol. 32, no. 5, pp. S112S119, 1998.

[2] J. Blacher, M. E. Safar, A. P. Guerin, B. Pannier, S. J. Marchais, and G. M. London, "Aortic pulse wave velocity index and mortality in end-stage renal disease," Kidney International, vol. 63, no. 5, pp. 1852-1860, 2003.

[3] G. M. London, A. P. Guérin, S. J. Marchais, F. Métivier, B. Pannier, and H. Adda, "Arterial media calcification in endstage renal disease: impact on all-cause and cardiovascular 
mortality," Nephrology Dialysis Transplantation, vol. 18, no. 9, pp. 1731-1740, 2003.

[4] F. Tentori, M. J. Blayney, J. M. Albert et al., "Mortality risk for dialysis patients With different levels of serum calcium, phosphorus, and PTH: the dialysis outcomes and practice patterns study (DOPPS)," American Journal of Kidney Diseases, vol. 52, no. 3, pp. 519-530, 2008.

[5] R. Wald, M. J. Sarnak, H. Tighiouart et al., "Disordered mineral metabolism in hemodialysis patients: an analysis of cumulative effects in the hemodialysis (HEMO) study," American Journal of Kidney Diseases, vol. 52, no. 3, pp. 531$540,2008$.

[6] R. Goetz, A. Beenken, O. A. Ibrahimi et al., "Molecular insights into the Klotho-dependent, endocrine mode of action of fibroblast growth factor 19 subfamily members," Molecular and Cellular Biology, vol. 27, no. 9, pp. 3417-3428, 2007.

[7] T. Yamashita, "Structural and biochemical properties of fibroblast growth factor 23," Therapeutic Apheresis and Dialysis, vol. 9, no. 4, pp. 313-318, 2005.

[8] M. Crestani, A. Sadeghpour, D. Stroup, G. Galli, and J. Y. L. Chiang, "Transcriptional activation of the cholesterol $7 \alpha$ hydroxylase gene (CYP7A) by nuclear hormone receptors," Journal of Lipid Research, vol. 39, no. 11, pp. 2192-2200, 1998.

[9] A. Kharitonenkov, T. L. Shiyanova, A. Koester et al., "FGF-21 as a novel metabolic regulator," Journal of Clinical Investigation, vol. 115, no. 6, pp. 1627-1635, 2005.

[10] M. Riminucci, M. T. Collins, N. S. Fedarko et al., "FGF23 in fibrous dysplasia of bone and its relationship to renal phosphate wasting," Journal of Clinical Investigation, vol. 112, no. 5, pp. 683-692, 2003.

[11] A. Khosravi, C. M. Cutler, M. H. Kelly et al., "Determination of the elimination half-life of fibroblast growth factor-23," Journal of Clinical Endocrinology and Metabolism, vol. 92, no. 6, pp. 2374-2377, 2007.

[12] H. Kurosu, Y. Ogawa, M. Miyoshi et al., "Regulation of fibroblast growth factor-23 signaling by Klotho," The Journal of Biological Chemistry, vol. 281, no. 10, pp. 6120-6123, 2006.

[13] T. Nakatani, B. Sarraj, M. Ohnishi et al., "In vivo genetic evidence for klotho-dependent, fibroblast growth factor 23 (Fgf23) -mediated regulation of systemic phosphate homeostasis," The FASEB Journal, vol. 23, no. 2, pp. 433-441, 2009.

[14] M. S. Razzaque, D. Sitara, T. Taguchi, R. St-Arnaud, and B. Lanske, "Premature aging-like phenotype in fibroblast growth factor 23 null mice is a vitamin D-mediated process," The FASEB Journal, vol. 20, no. 6, pp. 720-722, 2006.

[15] S. Liu, L. Vierthaler, W. Tang, J. Zhou, and L. D. Quarles, "FGFR3 and FGFR4 do not mediate renal effects of FGF23," Journal of the American Society of Nephrology, vol. 19, no. 12, pp. 2342-2350, 2008.

[16] E. G. Farrow, S. I. Davis, L. J. Summers, and K. E. White, "Initial FGF23-mediated signaling occurs in the distal convoluted tubule," Journal of the American Society of Nephrology, vol. 20, no. 5, pp. 955-960, 2009.

[17] K. I. Miyamoto, H. Segawa, M. Ito, and M. Kuwahata, "Physiological regulation of renal sodium-dependent phosphate cotransporters," Japanese Journal of Physiology, vol. 54, no. 2, pp. 93-102, 2004.

[18] K. I. Miyamoto, M. Ito, M. Kuwahata, S. Kato, and H. Segawa, "Inhibition of intestinal sodium-dependent inorganic phosphate transport by fibroblast growth factor 23," Therapeutic Apheresis and Dialysis, vol. 9, no. 4, pp. 331-335, 2005.

[19] M. Ito, Y. Sakai, M. Furumoto et al., "Vitamin D and phosphate regulate fibroblast growth factor-23 in K-562 cells,"
American Journal of Physiology, vol. 288, no. 6, pp. E1101E1109, 2005.

[20] O. I. Kolek, E. R. Hines, M. D. Jones et al., " $1 \alpha, 25$-dihydroxyvitamin D upregulates FGF23 gene expression in bone: the final link in a renal-gastrointestinal-skeletal axis that controls phosphate transport," American Journal of Physiology, vol. 289, no. 6, pp. G1036-G1042, 2005.

[21] D. M. Antoniucci, T. Yamashita, and A. A. Portale, "Dietary phosphorus regulates serum fibroblast growth factor-23 concentrations in healthy men," Journal of Clinical Endocrinology and Metabolism, vol. 91, no. 8, pp. 3144-3149, 2006.

[22] S. A. M. Burnett, S. C. Gunawardene, F. R. Bringhurst, H. Jüppner, H. Lee, and J. S. Finkelstein, "Regulation of Cterminal and intact FGF-23 by dietary phosphate in men and women," Journal of Bone and Mineral Research, vol. 21, no. 8, pp. 1187-1196, 2006.

[23] N. Ito, S. Fukumoto, Y. Takeuchi et al., "Effect of acute changes of serum phosphate on fibroblast growth factor (FGF) 23 levels in humans," Journal of Bone and Mineral Metabolism, vol. 25, no. 6, pp. 419-422, 2007.

[24] T. Krajisnik, P. Björklund, R. Marsell et al., "Fibroblast growth factor-23 regulates parathyroid hormone and $1 \alpha$-hydroxylase expression in cultured bovine parathyroid cells," Journal of Endocrinology, vol. 195, no. 1, pp. 125-131, 2007.

[25] T. Shimada, H. Hasegawa, Y. Yamazaki et al., "FGF-23 is a potent regulator of vitamin D metabolism and phosphate homeostasis," Journal of Bone and Mineral Research, vol. 19, no. 3, pp. 429-435, 2004.

[26] T. Kawata, Y. Imanishi, K. Kobayashi et al., "Parathyroid hormone regulates fibroblast growth factor- 23 in a mouse model of primary hyperparathyroidism," Journal of the American Society of Nephrology, vol. 18, no. 10, pp. 2683-2688, 2007.

[27] S. Liu, W. Tang, J. Zhou et al., "Fibroblast growth factor 23 is a counter-regulatory phosphaturic hormone for vitamin D," Journal of the American Society of Nephrology, vol. 17, no. 5, pp. 1305-1315, 2006.

[28] R. Marsell, E. Grundberg, T. Krajisnik et al., "Fibroblast growth factor-23 is associated with parathyroid hormone and renal function in a population-based cohort of elderly men," European Journal of Endocrinology, vol. 158, no. 1, pp. 125129, 2008.

[29] M. Roos, J. Lutz, H. Salmhofer et al., "Relation between plasma fibroblast growth factor-23, serum fetuin-A levels and coronary artery calcification evaluated by multislice computed tomography in patients with normal kidney function," Clinical Endocrinology, vol. 68, no. 4, pp. 660-665, 2008.

[30] O. Gutierrez, T. Isakova, E. Rhee et al., "Fibroblast growth factor-23 mitigates hyperphosphatemia but accentuates calcitriol deficiency in chronic kidney disease," Journal of the American Society of Nephrology, vol. 16, no. 7, pp. 2205-2215, 2005.

[31] T. Larsson, U. Nisbeth, Ö. Ljunggren, H. Jüppner, and K. B. Jonsson, "Circulating concentration of FGF-23 increases as renal function declines in patients with chronic kidney disease, but does not change in response to variation in phosphate intake in healthy volunteers," Kidney International, vol. 64, no. 6, pp. 2272-2279, 2003.

[32] D. Fliser, B. Kollerits, U. Neyer et al., "Fibroblast Growth Factor 23 (FGF23) predicts progression of chronic kidney disease: the mild to moderate kidney disease (MMKD) study," Journal of the American Society of Nephrology, vol. 18, no. 9, pp. 2600-2608, 2007. 
[33] Y. Imanishi, M. Inaba, K. Nakatsuka et al., "FGF-23 in patients with end-stage renal disease on hemodialysis," Kidney International, vol. 65, no. 5, pp. 1943-1946, 2004.

[34] N. Koh, T. Fujimori, S. Nishiguchi et al., "Severely reduced production of klotho in human chronic renal failure kidney," Biochemical and Biophysical Research Communications, vol. 280, no. 4, pp. 1015-1020, 2001.

[35] O. M. Gutiérrez, M. Mannstadt, T. Isakova et al., "Fibroblast growth factor 23 and mortality among patients undergoing hemodialysis," The New England Journal of Medicine, vol. 359, no. 6, pp. 584-592, 2008.

[36] M. A. I. Mirza, A. Larsson, L. Lind, and T. E. Larsson, "Circulating fibroblast growth factor- 23 is associated with vascular dysfunction in the community," Atherosclerosis, vol. 205, no. 2, pp. 385-390, 2009.

[37] M. A. I. Mirza, T. Hansen, L. Johansson et al., "Relationship between circulating FGF23 and total body atherosclerosis in the community," Nephrology Dialysis Transplantation, vol. 24, no. 10, pp. 3125-3131, 2009.

[38] G. Jean, J. C. Terrat, T. Vanel et al., "High levels of serum fibroblast growth factor (FGF)-23 are associated with increased mortality in long haemodialysis patients," Nephrology Dialysis Transplantation, vol. 24, no. 9, pp. 2792-2796, 2009.

[39] M. Inaba, S. Okuno, Y. Imanishi et al., "Role of fibroblast growth factor-23 in peripheral vascular calcification in nondiabetic and diabetic hemodialysis patients," Osteoporosis International, vol. 17, no. 10, pp. 1506-1513, 2006.

[40] O. M. Gutiérrez, J. L. Januzzi, T. Isakova et al., "Fibroblast growth factor 23 and left ventricular hypertrophy in chronic kidney disease," Circulation, vol. 119, no. 19, pp. 2545-2552, 2009.

[41] G. Jean, E. Bresson, J. C. Terrat et al., "Peripheral vascular calcification in long-haemodialysis patients: associated factors and survival consequences," Nephrology Dialysis Transplantation, vol. 24, no. 3, pp. 948-955, 2009.

[42] Q. W. Pan, R. X. Ruan, D. A. Wheeler et al., "Fibroblast growth factor-23 inhibits inflammation-induced calcification in vitro in human vascular smooth muscle cells," ASN Renal Week, 2009.

[43] F. Saji, K. Shiizaki, S. Shimada et al., "Regulation of fibroblast growth factor 23 production in bone in uremic rats," Nephron. Physiology, vol. 111, no. 4, pp. 59-66, 2009.

[44] O. Gutierrez, T. Isakova, E. Rhee et al., "Fibroblast growth factor-23 mitigates hyperphosphatemia but accentuates calcitriol deficiency in chronic kidney disease," Journal of the American Society of Nephrology, vol. 16, no. 7, pp. 2205-2215, 2005.

[45] S. Pande, C. S. Ritter, M. Rothstein et al., "FGF-23 and sFRP4 in chronic kidney disease and post-renal transplantation," Nephron-Physiology, vol. 104, no. 1, pp. p23-p32, 2006.

[46] G. A. Block, T. E. Hulbert-Shearon, N. W. Levin, and F. K. Port, "Association of serum phosphorus and calcium x phosphate product with mortality risk in chronic hemodialysis patients: a national study," American Journal of Kidney Diseases, vol. 31, no. 4, pp. 607-617, 1998.

[47] G. A. Block, P. S. Klassen, J. M. Lazarus, N. Ofsthun, E. G. Lowrie, and G. M. Chertow, "Mineral metabolism, mortality, and morbidity in maintenance hemodialysis," Journal of the American Society of Nephrology, vol. 15, no. 8, pp. 2208-2218, 2004.

[48] K. Kalantar-Zadeh, N. Kuwae, D. L. Regidor et al., "Survival predictability of time-varying indicators of bone disease in maintenance hemodialysis patients," Kidney International, vol. 70, no. 4, pp. 771-780, 2006.

[49] M. L. Melamed, J. A. Eustace, L. Plantinga et al., "Changes in serum calcium, phosphate, and PTH and the risk of death in incident dialysis patients: a longitudinal study," Kidney International, vol. 70, no. 2, pp. 351-357, 2006.

[50] M. Noordzij, J. C. Korevaar, E. W. Boeschoten, F. W. Dekker, W. J. Bos, and R. T. Krediet, "The kidney disease outcomes quality initiative (K/DOQI) guideline for bone metabolism and disease in CKD: association with mortality in dialysis patients," American Journal of Kidney Diseases, vol. 46, no. 5, pp. 925-932, 2005.

[51] Y. Slinin, R. N. Foley, and A. J. Collins, "Calcium, phosphorus, parathyroid hormone, and cardiovascular disease in hemodialysis patients: the USRDS waves 1, 3, and 4 study," Journal of the American Society of Nephrology, vol. 16, no. 6, pp. 1788-1793, 2005.

[52] L. A. Stevens, O. Djurdjev, S. Cardew, E. C. Cameron, and A. Levin, "Calcium, phosphate, and parathyroid hormone levels in combination and as a function of dialysis duration predict mortality: evidence for the complexity of the association between mineral metabolism and outcomes," Journal of the American Society of Nephrology, vol. 15, no. 3, pp. 770-779, 2004.

[53] P. Ravani, F. Malberti, G. Tripepi et al., "Vitamin D levels and patient outcome in chronic kidney disease," Kidney International, vol. 75, no. 1, pp. 88-95, 2009.

[54] R. Shroff, M. Egerton, M. Bridel et al., "A bimodal association of vitamin d levels and vascular disease in children on dialysis," Journal of the American Society of Nephrology, vol. 19, no. 6, pp. 1239-1246, 2008.

[55] M. Wolf, A. Shah, O. Gutierrez et al., "Vitamin D levels and early mortality among incident hemodialysis patients," Kidney International, vol. 72, no. 8, pp. 1004-1013, 2007.

[56] D. Medici, M. S. Razzaque, S. DeLuca et al., "FGF-23-Klotho signaling stimulates proliferation and prevents vitamin Dinduced apoptosis," Journal of Cell Biology, vol. 182, no. 3, pp. 459-465, 2008.

[57] M. S. Razzaque, "Does FGF23 toxicity influence the outcome of chronic kidney disease?" Nephrology Dialysis Transplantation, vol. 24, no. 1, pp. 4-7, 2009. 


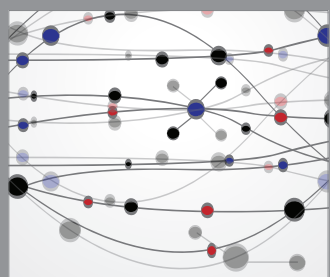

The Scientific World Journal
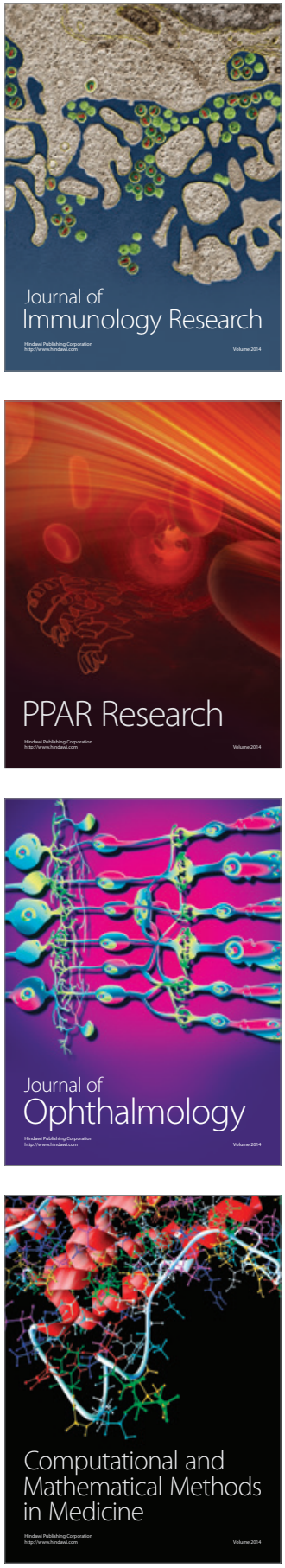

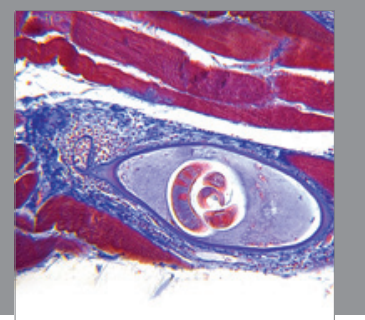

Gastroenterology

Research and Practice
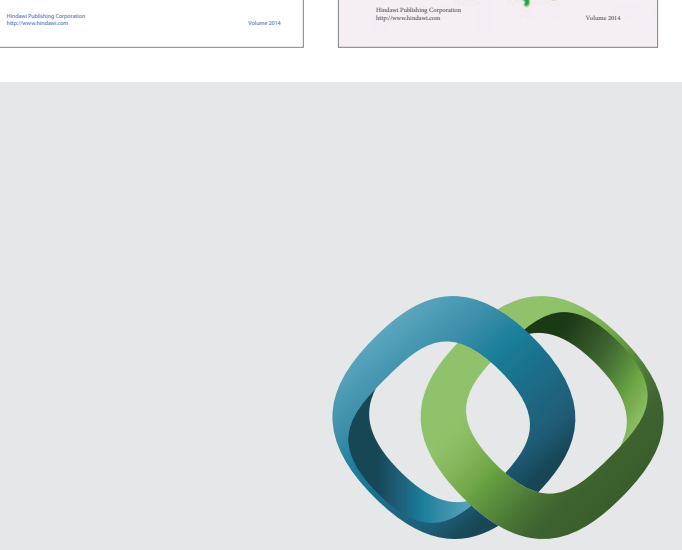

\section{Hindawi}

Submit your manuscripts at

http://www.hindawi.com
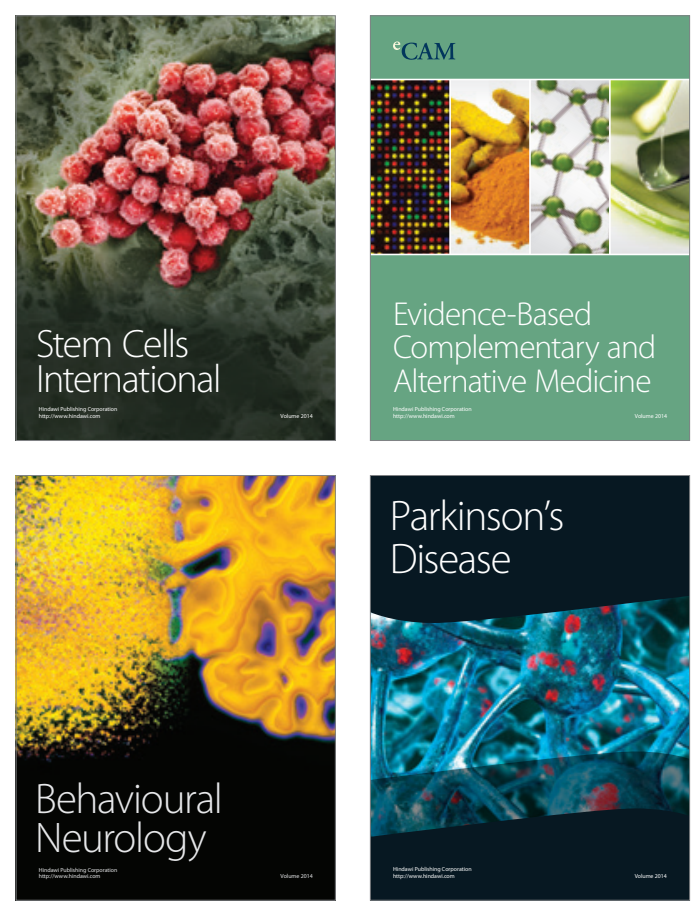

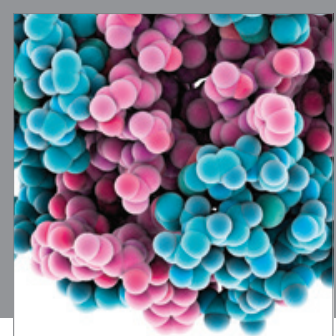

Journal of
Diabetes Research

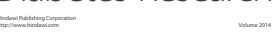

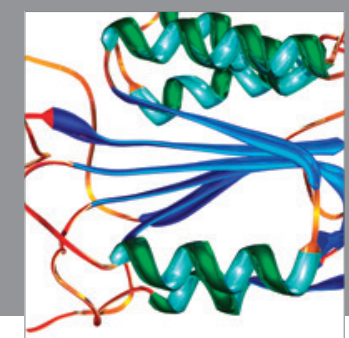

Disease Markers
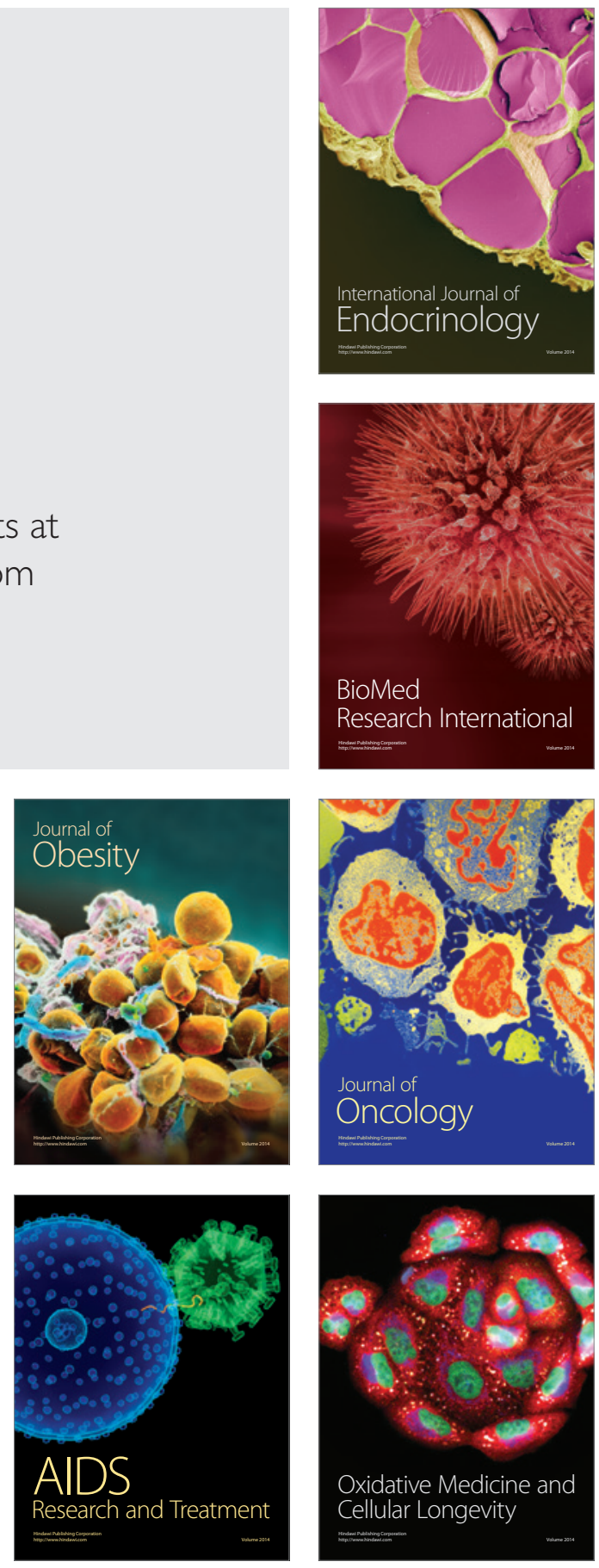